\title{
THE 1961 EXHIBITION OF THE INSTITUTE OF PHYSICS AND THE PHYSICAL SOCIETY
}

T HE forty-fifth Annual Exhibition of Scientific Instruments and Apparatus held in the Old and New Halls of the Royal Horticultural Society in London during January 16-21 was the first since the amalgamation of the Institute and the Society. By continuing in its established tradition, it has incidentally managed to conform with ancient precedent, for the sixth King James of Scotlend also suffered no change of character on becoming the first of a united body. It was propitious that the activities and publications of the Institute and Society were displayed conspicuously on an attractively arranged stand, and many visitors must have been glad of this opportunity to make a closer acquaintance with their host. From the point of view of leaming about new developments, it was a very good exhibition. The footnote 'demonstration' in the catalogue usually meant not just that a device would be working, but that an experiment to explain its principle or its uses had been mounted. Things progress so fast nowadays that even the term 'trend' would be out of place (if it has not already been registered to name a detergent, anyway); and the uninitiated can only hope to sense what is more like a rate of increase of acceleration. This seemed steepest in fast-operating elements for computers, instrumentation for thermonuclear research, and micro-wave amplifiers, all directed towards the future; and in physics applied to archæology, using nuclear and other techniques for raking up the past.

Several stands exhibited devices incorporating the new Esaki or 'tunnel' diode, made of germanium antimonide or indium antimonide; this operates by a quantum-mechanical tunnelling across the energy gap at a $p-n$ junction, and its chief virtue is high speed. Its uses in logic and memory circuits and for amplitude-time discrimination were shown. The Royal Radar Establishment illustrated the behaviour of a tunnel diode in a magnetic field, which alters the energy gap and reduces the current. Microminiaturized solid circuits, and also circuits built up from thin films, were displayed. Among new ceramic materials were those designed for capacitors, high. temperature ceramics of the silicon nitride and silicon carbide type, and ceramic elements for electroluminescent panels.

Thin magnetic films, using material of the permalloy type, are made for information storage and millimicrosecond switching elements. Two firms demonstrated apparatus for determining the hysteresis loops of thin films, and for observing the domain boundaries visually by means of the Kerr magnoto-optical effect. The General Electric Company showed how the easy direction of magnetization can be determined, and also a device for testing magnetic-film matrix elements under simulated computer conditions.

How to deal with the $14-\mathrm{MeV}$. neutrons resulting from the $(\mathrm{D}, \mathrm{T})$ reaction is one of the problems of the thermonuclear investigators, and threo neutron generators producing $14-\mathrm{MeV}$. neutrons by bom. barding tritium-containing targets with $100-\mathrm{kV}$. accelerated deuterons were displayed, for either pulsed or steady operation. These will enable studies of this interesting product of the $(D, T)$ reaction to be made at high flux densities without having to operate Sceptre and such-like as sources. Associated Electrical Industries, Ltd., of Aldermaston, showed a data-processing system for thermonuclear research, following the fluctuations of an unstabilized discharge in a glass torus, intended to study what goes on in Sceptre itself.

Amplification of various kinds was featured in many exhibits. Five-stage optical image intensifiers, using secondary emission from thin-film dynodes and amplifying up to 100,000 times, represent one aspect ; and there were many micro-wave low-noise and other amplifiers. Standard Telephones and Cables, Ltd., illustrated the principle of the parametric amplifier by a mechanical analogue; and there was a parametric amplifier for ultra-high frequencies which operated after frequency conversion down to the $X$-band. I learned from a physicist working in this field that the maser of the future for satellite and space work is the travelling-wave tube-of the immediate future, that is. Production types of travellingwave tubes were shown, and the Services Electronics Research Laboratories had a high-power version which operated at about $5 \mathrm{~kW}$. This establishment also had a cyclotron wave-amplifier (Adler tube); and Associated Electrical Industries, Ltd., featured what was called a quadrupole electrostatic cyclotron wave-amplifier, which has not so far reached the stage of being a low-noise device.

Fundamental physics was exhibited in the demonstration arranged by the Atomic Energy Research Establishment of the Mössbauer effect, in which the $\gamma$-rays emitted by one transition in an excited iron-57 nucleus are absorbed by a different transition in an iron-57 target moving at a suitable relative velocity, the resonance being displayed on a velocity spectrometer. Among the exhibits from the National Physical Laboratory was a new absolute radiometer, determining radiant power in terms of electrical power. What might almost be called 'unfundamental physics' was shown in a piece of intriguing hydromotry, which used a thin-walled transducer vibrating within a liquid to determine the density of the liquid to better than 1 per cent-valuable for the particular kind of instrumentation that it was aimed to do, of course, but a long way round in principle.

Magnetometry was represented by proton-resonance magnetometers ; one by Mullard, Ltd., adapted to control the oscillator at what the description consistently and nostalgically called the "Lamour frequency", and another as part of the comprehensive exhibit of the Research. Laboratory for Archæology and the History of Art, Oxford, where one would have liked to spend a good deal longor learning about the highly ingenious stratagems used to detect buried buildings and pottery by measuring the small changes they produce in the ambient magnetic field and its vertical gradient; it was amazing to learn that the real source of this change is in many cases the result 
of a slight alteration in the magnetic properties of clay produced by firing. The Signals Research and Development Establishment demonstrated a rubidium-vapour magnetometer, which is best described as the optical-pumping (or 'photon-spin pumping') device working in reverse. The effect of a magnetic field, which separates the otherwise degenerate spinlevels by an extent proportional to this field if it is small, is used to measure the strength of the field. A perpendicular radio-frequency magnetic field is adjusted to the energy difference between the pair of levels which have been population-inverted (that is, to the Larmor frequency when the system starts to operate maser-wise, a state that is detected by the strong absorption of the pumping beam of circularly polarized rubidium $D_{1}$ light). Thus, instead of tuning to a given radio-frequency by altering the field, the system is tuned to the ambient field by altering the frequency. This is sufficiently sensitive for geomagnetic observations, and may possibly be applied to aerial and space magnetic surveys; indeed, the actual apparatus was too sensitive to work in the disturbing fields of the Hall, and was planted out on the Westminster School playing-field.
These are the impressions of a day's visit; a proper appreciation would have needed the full five days, and some considerable preparation beforehand. There was much that I missed, and one highlight in the form of a miniature lighthouse with a brilliant source that I even failed to identify properly. The exhibits from various research laboratories of the Department of Scientific and Industrial Research were representative of the wide variety of tasks they undertake. The Science Museum had two exhibits, one ancient and one modern, and there were six contributions from university departments ; it would add further facets to a brilliant occasion if we could see, among all the new tools for research and development, more of the new apparatus designed at the universities for their practical classes and demonstrations, and at the Science Museum for the instruction of the general public.

Evening discourses were given during the Exhibition by F. S. Burt, on "Hydrodynamics Research"; by Dr. G. E. R. Deacon, on "The Physics of the Ocean"; and by Dr. F. P. Bowden, on "Some Physical Problems in Travelling at Supersonic Speed". G. R. NOAKES

\section{CALORIE BALANCE IN MAN}

T HE one hundred and thirty-seventh meeting of the Nutrition Society was held in the Middlesex Hospital Medical School on December 17, and took the form of a symposium on "Calorie Balance in Man". Perhaps it was optimistic to expect a symposium, continuing from 10.15 a.m. to 4.30 p.m. on the Satur. day a week before Christmas, to be popular. In fact, it was a well-attended meeting with an audience of more than 100. The chairman was Sir Charles Dodds.

Not so many years ago the subject of "Calorie Balance" would have been an unlikely topic for such a symposium. Interest in calories, at least in relation to human energy, was at a low ebb scientifically and a rather naive idea was prevalent that little new remained to be discovered in this field. Far from this being the case, a most notable impression gained from the speakers at this symposium was the great dearth of reliable relevant information.

The lack of information and the misconception about many aspects of human energy metabolism were mentioned in relation to some of the "Basic Physiological Factors affecting Calorie Balance", the title of the paper by Dr. J. V. G. A. Durnin (University of Glasgow). Part of the complexity of 'calorie balance' arises because of the difficulty of assessing methodological accuracy; there is no sure way of knowing whether the intake of calories or the expenditure of energy represents the 'normal' or usual state for the individual subject. $\mathrm{He}$, or she, may be eating more or less, during the experimental study, than would normally be the case, or the level of energy expenditure may be altered. Since it is extremely difficult under field conditions to carry out such measurements over a period of more than a few days, theoretically one possible way of finding a departure from the normal would be to measure a change of weight in the subject. Part of this paper and a large part of the discussion were devoted to the balance of weight, and Dr. Durnin showed results for young men, weighed under highly stan- dardized conditions, where their gross body-weight changed by up to $3 \mathrm{lb}$. (1.4 kgm.) from day to day. Such changes, presumably almost entirely due to alterations in the fluid content of the body, make the weighing of subjects quite worthless in regard to the balance of energy.

Other factors dealt with were exercise (so often discussed emotionally rather than rationally), climate, age (growth in the young and inversion of growth in the old) and the efficiency of utilization of food by the body.

That most of us do not normally attain balance of energy from day to day was shown clearly by an analysis on the results for 69 individuals, where only 4 subjects had a significant correlation between their intake and output of energy; our 'appetite' is a fickle mechanism.

Calorie balance is probably a long-term control, which may be effected by centres in the hypothalamus responding to changes in the fat depots of the body and then influencing the general level of food intake and perhaps even of bodily activity. In this control, the reticular activating system of the brain stem, the limbic system and the hypothalamus may act together. This was suggested by Dr. G. C. Kennedy (University of Cambridge) in his paper "Central Nervous Regulation of Calorie Balance". The importance of exercise was stressed, both in relation to man and to studies on animals; much work on rats or mice is not comparable because of varying levels of physical activity resulting from restrictions imposed by some types of animal cages. Similarities between a 42 stone (267 kgm.) obese patient and experimentally obese rats were shown by both the astonishing hyperphagia and also lack of resentment or discomfort when food was restricted. They were also alike in their inactivity.

It has been the custom, at certain levels of medical therapeutics, to treat obesity as if it were primarily an endocrine disorder and give the patient mixtures of tissue extracts from various endocrine glands. 